\title{
Integrin $\alpha 2$-deficient mice provide insights into specific functions of collagen receptors in the kidney
}

\author{
Rainer Girgert ${ }^{1}$, Maria Martin ${ }^{1}$, Jenny Kruegel ${ }^{2}$, Nicolai Miosge², Johanna Temme ${ }^{1}$, Beate Eckes ${ }^{3}$, \\ Gerhard-Anton Müller', Oliver Gross ${ }^{1 *}$
}

\begin{abstract}
Background: Integrins are important cellular receptors for collagens. Within the glomerulus, podocytes regulate the integrity of the glomerular basement membrane (GBM) by sensing the presence of collagen and regulating collagen IV synthesis. The present study evaluates the role of integrin $\alpha 2$ (ITGA2) in cell-matrix interaction.

Methods and Results: ITGA2-deficient mice had normal renal function but moderate proteinuria and enhanced glomerular and tubulointerstitial matrix deposition. Electron microscopy demonstrated irregular podocyte-matrix interaction, causing pathological protrusions towards the urinary (podocyte) side of the GBM. These characteristic subepithelial bulges mimic the renal phenotype of mice, which are deficient in another collagen receptor, discoidin domain receptor (DDR)1. Using immunogold staining, ITGA2 expression was found to localize to the basolateral site of the podocyte foot processes. ITGA2-deficient mice overexpressed transforming growth factor (TGF) $\beta$ and connective tissue growth factor (CTGF) compared with wild-type mice. Using in situ hybridization, tubular cells were found to be the primary site of TGF $\beta$ synthesis and podocytes the source of CTGF in ITGA2deficient mice.
\end{abstract}

Conclusion: These findings support our hypothesis that both these collagen receptors (ITGA2 and DDR1) play a similar role within the kidney. Further, cell-matrix interaction via collagen receptors seems to be crucial for maintenance of normal GBM architecture and function. Targeting collagen receptors such as ITGA2 might be a new form of treatment for progressive fibrotic diseases.

\section{Introduction}

Integrins are cellular receptors that are important for sensing the extracellular environment and adjacent cells. Each cell type expresses a characteristic combination of $\alpha$ - and $\beta$-subunits. The $\alpha$-subunit of an integrin predicts its ligand specificity, whereas the cytoplasmic tail of the $\beta$-subunit interacts with the cytoskeleton and with proteins involved in signal transduction. Integrin $\alpha 1$ has only one known partner, $\beta 1 ; \alpha 1 \beta 1$ is an important receptor for collagens and laminins in basement membranes $[1,2]$. The most abundant integrins within the glomerulus are $\alpha 1 \beta 1, \alpha 2 \beta 1$ and $\alpha 3 \beta 1$. Integrin $\alpha 1 \beta 1$ is expressed in all glomerular cell types [3], and plays a

\footnotetext{
* Correspondence: gross.oliver@med.uni-goettingen.de

'Department of Nephrology and Rheumatology, Georg-August-University Goettingen, Goettingen, Germany

Full list of author information is available at the end of the article
}

crucial role in cell proliferation and collagen turnover, and in sensing extracellular collagen levels and downregulating endogenous collagen synthesis [4]. $\alpha 1 \beta 1$ integrin is expressed primarily on mesangial cells, $\alpha 2 \beta 1$ primarily on endothelial cells, and $\alpha 3 \beta 1$ on podocytes [5]. Integrin $\alpha 1$-knockout mice are fertile with no overt phenotype [5-7], whereas targeted deletions of $\alpha 4$ and $\alpha 5$ integrins are lethal during embryogenesis [8]. Embryonic fibroblasts derived from $\alpha 1$-deficient animals are unable to spread on collagen IV [9]. The role of $\alpha 1 \beta 1$ integrin in collagen-dependent cell proliferation, adhesion, matrix remodeling and mesangial cell migration points to integrins playing a role in the pathogenesis of collagen diseases such as the hereditary type IV collagen disease Alport syndrome (AS).

AS is caused by mutations in the $\alpha 3 / 4$ or 5 chains of type IV collagen, which result in proteinuria and renal 
failure [10]. Type IV collagen is the major constituent of the glomerular basement membrane (GBM). During embryogenesis, the premature GBM, consisting of $\alpha 1$ / $\alpha 1 / \alpha 2$ (IV) chains, is produced by endothelial cells and podocytes. The mature GBM, containing $\alpha 3 / \alpha 4 / \alpha 5$ (IV) chains, is built solely by podocytes [11-14]. Mutations in the $\alpha 3 / \alpha 4 / \alpha 5$ (IV) chains in AS result in characteristic ultrastructural changes in the GBM [10]. One important step in the pathogenesis of AS is thought to be an altered cell-matrix interaction via the podocyte collagen receptors. For example, ASmice, which carry an additional knockout for the integrin $\alpha 1$ gene, show delayed onset and slower disease progression [5].

Integrins can be activated by triple-helix type I and IV collagens [15]. Binding of integrin to its ligands influences the actin cytoskeleton via activation of cdc42, Rac1 and focal adhesion kinase (FAK) [16]. The activation of $\alpha 1 \beta 1$ integrin downregulates collagen synthesis [4], suggesting that the functional loss of this integrin could predispose the host to augmented sclerosis after injury. Transforming growth factor (TGF) $\beta$ is known to stimulate the production of the extracellular matrix (ECM) in podocytes [17]. As integrin-mediated FAK activation leads to the downregulation of TGF- $\beta$ receptors, loss of $\alpha 1 \beta 1$ function results in increased collagen expression [18].

The possible role of collagen receptors other than integrins, such as discoidin domain receptor (DDR) 1 in renal disease has been previously addressed by our group $[19,20]$. Loss of DDR1 delays renal fibrosis in AS [20]. The role of $\alpha 2 \beta 1$ integrin in renal pathology is less clear. Examination of integrin $\alpha 2$ knockout mice showed a multifaceted phenotype including defects of branching morphogenesis, hemostasis and a partially defective platelet interaction with collagen $[7,21]$. The nonlethal phenotype of the $\alpha 2$-deficient mice implies that many of the key functions of $\alpha 2 \beta 1$ can be mimicked by other integrins. In the present study, we analyzed the renal phenotype of integrin $\alpha 2$-deficient mice, and found that loss of integrin $\alpha 2$ results in irregular podocyte-matrix interaction, causing pathological protrusions towards the urinary (podocyte) side of the GBM and increased TGF $\beta$ and connective tissue growth factor (CTGF) expression.

\section{Materials and methods}

The animal care and experiments were performed according to the Declaration of Helsinki and the Guide for the Care and Use of Laboratory Animals (NIH).

\section{Knockout mice}

The methods for generation of integrin $\alpha 2$ knockout mice and for PCR-based genotyping have been described previously $[7,21]$. The ITGA2 knockout and wild-type mice (provided by B. Eckes, University of Cologne, Cologne, Germany) used here were bred on a C57Bl6 background under pathogen-free housing conditions at the local animal facility with a 12-h light/dark cycle and unlimited access to food and water. The DDR1 ${ }^{-1-}$ mice were provided by W. Vogel, University of Toronto, Toronto, Canada, and the collagen (IV) $\alpha 3$ (COL4A3) $)^{-1-}$ mice by Jackson Immunoresearch Laboratories (Westgrove, PA, USA). COL4A3 ${ }^{-1-}$ mice were crossbred to the C57Bl6 background for nine generations. No animals were lost due to infections during monitoring. For western blotting, histology and assessment of urea levels, animals were examined at 100 and 150 days of age.

\section{Western blot analysis}

Three to four kidneys from either the wild-type or ITGA2-deficient groups were homogenized in a solution of Tris-buffered saline (TBS) containing a cocktail of protease inhibitors (phenylmethylsulfonyl fluoride (PMSF), leupeptin and pepstatin). Next, $20 \mu \mathrm{g}$ of protein (equal loading dose shown by the bicinchoninic acid (BCA) protein assay) was solubilized in lithium dodecyl sulfate (LDS) sample buffer and separated in a 4 to $12 \%$ gel (NuPage Novex Bis-Tris Gel; Invitrogen, Carlsbad, CA, USA), transferred to a polyvinylidene fluoride membrane, and blocked with $5 \%$ dry milk powder in TBS with Triton X-100 (TBST). The membrane was incubated overnight with mouse anti-TGF $\beta 1$ (1:2000; R\&D Systems, Minneapolis, USA) and rabbit anti-CTGF (1:2000; Abcam Limited, Cambridge, United Kingdom), then with the horseradish peroxidase-conjugated secondary antibody (Dako, Hamburg, Germany) for 1 hour at room temperature. The blots were developed by chemoluminescence using enhanced chemiluminescence (GE-Healthcare, Freiburg, Germany). Protein expression was analyzed by densitometry (Kd1 software; Kodak, Rochester, NY, USA).

\section{In situ hybridization}

Paraffin-embedded sections ( $5 \mu \mathrm{m}$ thick) were dewaxed and rehydrated in decreasing concentrations of ethanol. After two washes in phosphate-buffered saline (PBS), sections were partially digested with $6 \mu \mathrm{g} / \mathrm{ml}$ proteinase K (Qiagen, Hilden, Germany) for $30 \mathrm{~min}$. Sections were post-fixed with $4 \%$ formaldehyde for 5 minutes before hybridization with a digoxigenin (DIG)-labeled RNA probe specific for either TGF $\beta$ mRNA or CTGF mRNA. Probes for in situ hybridization were labeled with DIG by in vitro transcription in the presence of DIG-UTP using reverse transcriptase PCR products of TGF $\beta$ and CTGF as templates, linked to promoters of SP6 or T7 RNA polymerase by molecular cloning. DIG-labeled probes were hybridized to the sections in the hybridization solution at $42^{\circ} \mathrm{C}$ overnight. Subsequently, sections were washed twice in $2 \times$ sodium citrate buffer and 
PBS. Bound probes were detected with (1:500) rabbit anti-DIG-AP (Roche, Penzberg, Germany) in TBS containing 5\% dry milk powder, and visualized using the diluted alkaline phosphatase substrate nitro blue tetrazolium chloride/5-bromo-4-chloro-3'-indolyphosphate p-toluidine salt (NBT/BCIP) (1:50; Roche, Penzberg, Germany) at $\mathrm{pH} 9.5$.

\section{Immunohistochemistry of tissue sections and scoring of fibrosis}

From each group, three to five mice were killed and transcardially perfused with a solution of paraformaldehyde and glutaraldehyde; kidneys were immersion fixed as described previously [8]. Semi-thin and thin sections of paraffin wax-embedded kidney were cut (Ultracut UCT ultramicrotome; Reichert Inc., Depew, NY, USA) into sections $5 \mu \mathrm{m}$ thick, then dewaxed, rehydrated in graded alcohol and blocked with $5 \%$ bovine serum albumin (BSA) in TBS at room temperature. Endogenous peroxidases were blocked by treatment with $3 \%$ hydrogen peroxide in PBS for 10 minutes. Primary antibodies (rabbit anti-mouse EHS-laminin and rabbit anti-mouse fibronectin, 1:1000; gift from M. Paulsson, Cologne, Germany) were incubated overnight at $4^{\circ} \mathrm{C}$. Next, sections were incubated with goat anti-rabbit Cy3 (Jackson ImmunoResearch Laboratories Inc.) as a secondary antibody and analyzed under a microscope (Axiophot; Zeiss, Göttingen, Germany). Stained sections were scored for protein deposition as described previously [10]. Glomerulosclerosis was defined as the loss of $>50 \%$ of the glomerular lumen due to matrix accumulation. Tubulointerstitial fibrosis was evaluated in a similar manner by grading by a blinded observer of 12 kidney sections, each from three animals per group, for accumulation of ECM (graded as $0,1+$ and 2+).

\section{Electron microscopy and immunogold detection of integrin $\alpha 2$}

For ultrastructural electron microscopy, kidney samples $\left(4-\mathrm{mm}^{3}\right)$ taken from three mice per group were fixed in Karnovsky solution and embedded in epoxy resin as described previously [22]. Ultrathin sections were prepared (Reichert-Jung Ultracut E; Leica, Wetzlar, Germany) and examined under an electron microscope (LEO 906E; Zeiss, Oberkochen, Germany). For immunogold staining, kidney samples of $1 \mathrm{~mm}^{3}$ were fixed and embedded in a hydrophilic resin (LR-Gold; London Resin Company, Reading, UK) as described previously [23]. Ultrathin sections were blocked with $1 \%$ BSA in PBS for $20 \mathrm{~min}$ at room temperature. The primary antibody (rabbit anti-mouse integrin $\alpha 2,1: 50$ in PBS; Abcam) was incubated for 1 hour at room temperature. A secondary antibody (goat anti-rabbit IgG, 1:300 in PBS; Medac, Hamburg, Germany) was coupled to 16$\mathrm{nm}$ colloidal gold particles according to standard protocols [24] and incubated for $20 \mathrm{~min}$ at room temperature. Contrast staining with $1 \%$ uranyl acetate and lead citrate was carried out for $10 \mathrm{~min}$ each at room temperature. Negative control experiments were performed using ITGA2 $2^{-1-}$ kidneys and by treating the sections with $1 \%$ BSA instead of the primary antibody. To exclude binding of uncoupled gold particles to tissue structures, control sections were incubated with a pure colloidal gold solution.

\section{Analyses of urine and serum}

Proteins from $20 \mu \mathrm{l}$ of urine of three to five mice per group were precipitated using methanol/chloroform and dissolved in loading buffer. They were separated in a pre-cast 4 to $12 \%$ gradient polyacrylamide gel (Invitrogen) and stained with Coomassie blue, then the protein bands were densitometrically analyzed [19]. The serum urea levels of three $t$ five mice per group was determined using an automatic analyzer (Hitachi 91; Boehringer Mannheim, Germany).

\section{Statistics}

Results are presented S means and standard deviation. Data were analyzed by one-way analysis of variance (ANOVA).

\section{Results}

Loss of ITGA2 mimics the renal phenotype of DDR1 knockouts (ultrastructural changes in GBM)

Electron microscopy demonstrated local GBM thickening (Figure 1b, c, white arrowheads ) in ITGA2 knockouts. These local changes affected 1 to $3 \%$ of the total GBM, compared with $100 \%$ of the GBM affected in AS (Figure 1g-i) [10]. The protrusions in ITGA2 knockout micestained very homogeneously (Figure 1-c), similar to the protrusions found in DDR1 knockouts (Figure 1d-f) [14]. By contrast, the splitting of the GBM found in AS mice was much more heterogeneous and contained fibrillar collagens (Figure 1h, i). In AS mice, the GBM changes were accompanied by severe loss and effacement of the podocyte foot processes (Figure 1i), whereas the foot processes in ITGA2 knockout mice appeared to be normal and stayed attached to the GBM even in areas of GBM thickening (Figure 1b, c).

\section{Loss of ITGA2 causes moderate glomerular and tubulointerstitial damage}

According to our hypothesis, loss of ITGA2 may influence cell-matrix interactions, including profibrotic effects due to the upregulation of profibrotic cytokines. Compared with wild-type controls (Figure 2a, b), 150day-old ITGA2 ${ }^{-1-}$ mice (Figure 1e, f) showed minor glomerular, periglomerular and tubulointerstitial matrix accumulation. In parallel, total kidney extracts from 


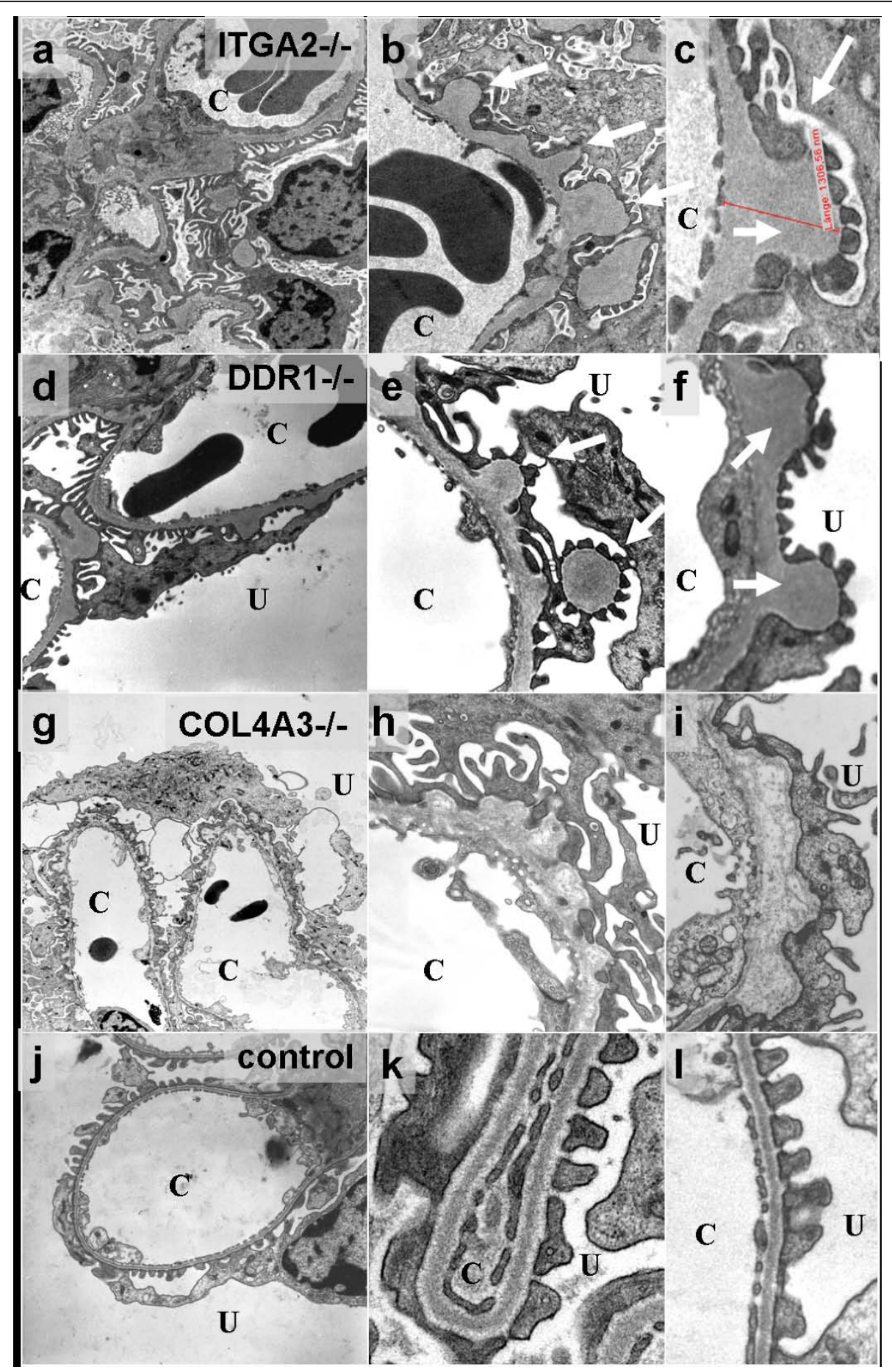

Figure 1 Ultrastructural analysis of the glomerular basement membrane. Mice deficient in (a-c) integrin $\alpha 2$ (ITGA2), (d-f) discoidin domain receptor (DDR)1 and ( $\mathbf{g}-\mathbf{i})$ collagen (IV) $\alpha 3$ (COL4A3), and (j-l) wildtype controls. Electron microscopy demonstrates a localized, mushroom-like isodense thickening of the glomerular basement membrane (GBM) in (a-c) ITGA2-deficient mice, compared with (j-l) wild-type mice. (b, $\mathbf{c})$ These characteristic subepithelial bulges (white arrows) seem to be identical to those observed in (d-f) DDR1 knockouts [19]) (g-i) By contrast, splitting and thickening of the GBM and fibrillar collagens indicating fibrosis were found in COL4A3-deficent mice. Original magnification: $(\mathbf{a}, \mathbf{d}, \mathbf{g}, \mathbf{j}) \times$ 3,000 to 7,$000 ;(\mathbf{b}, \mathbf{c}, \mathbf{e}, \mathbf{f}, \mathbf{h}, \mathbf{i}, \mathbf{k}, \mathbf{I}) \times 15,000$ to 30,000 . $U$ = urinary space; $C=$ capillary lumen. 


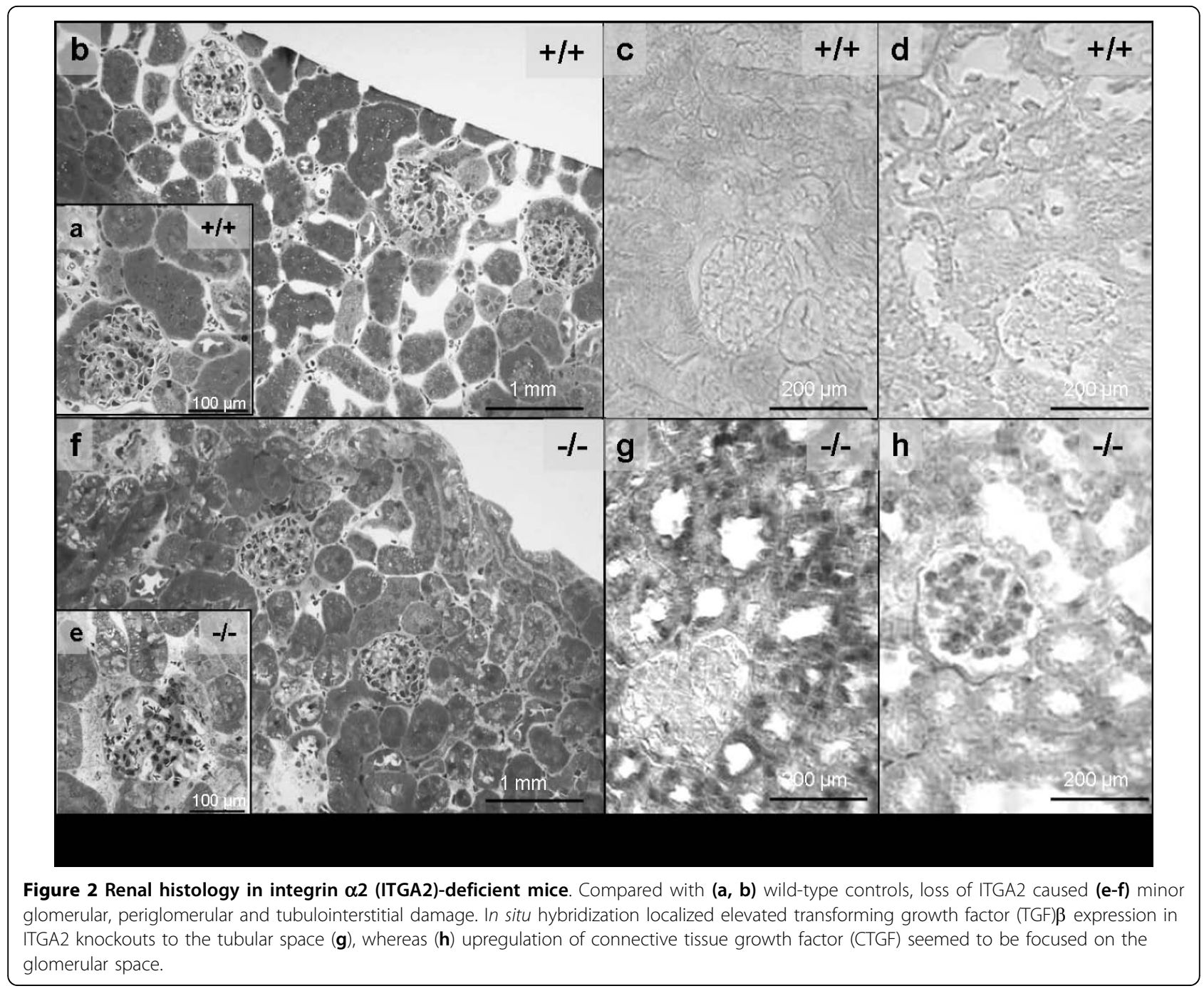

ITGA2 $2^{-1-}$ mice showed upregulation of TGF $\beta$ (Figure 3, lanes 2 and 3), whereas TGF $\beta$ in wild-type mice was barely detectable (Figure 3, lane 1). Additionally, the signal for CTGF was increased in ITGA2 knockouts (Figure $2 \mathrm{~d}$ ) compared with wild-type mice. Densitometric evaluation of the TGF $\beta$ bands revealed 17.3-fold (100 days) and 14.6-fold (150 days) increases in TGF $\beta$ and 2.1-fold and 3.6-fold increases in CTGF in ITGA2 knockout mice (Figure 3).

In situ hybridization of TGF $\beta$ and CTGF confirmed these results (Figure 2c versus Figure $2 \mathrm{~g}$ and Figure $2 \mathrm{~d}$ versus Figure 2h). TGF $\beta$ was not detectable in the kidneys of wild-type mice, whereas in ITGA2 knockouts, TGF $\beta$ was primarily expressed in tubular cells, with a weak glomerular signal (Figure 2g). Kidneys of wild-type mice were also negative for CTGF, but in ITGA2 knockouts, expression of CTGF was more pronounced in the glomerulus than in tubular cells (Figure 2h).
Loss of ITGA2 increases glomerular and global tubulointerstitial matrix deposition and fibrosis

To analyze the effects of ITGA2 knockout on the production of ECM and induction of fibrosis, kidney sections of healthy (Figure 4a) and knockout mice (Figure 4b, c) were immunostained for EHS laminin (laminin1), a marker for global glomerular and tubulointerstitial ECM deposition. The glomerular and tubulointerstitial matrix deposition was somewhat increased in 100-day-old ITGA2 ${ }^{-1-}$ mice (Figure $4 \mathrm{~b}$ ) compared with wild-type controls (Figure 4a), and this difference was increased further in 150-day-old mice (Figure 4c). Increased laminin-1 synthesis in the GBM of ITGA2 $2^{-1-}$ mice is shown at higher magnification in Figure 4 (b, c, insets)

Fibronectin staining also demonstrated moderate glomerular, periglomerular and tubulointerstitial deposition in 150-day-old ITGA2 $2^{-1-}$ mice (Figure 4e) compared with wild-type controls (Figure 4d). The tubulointerstitial 


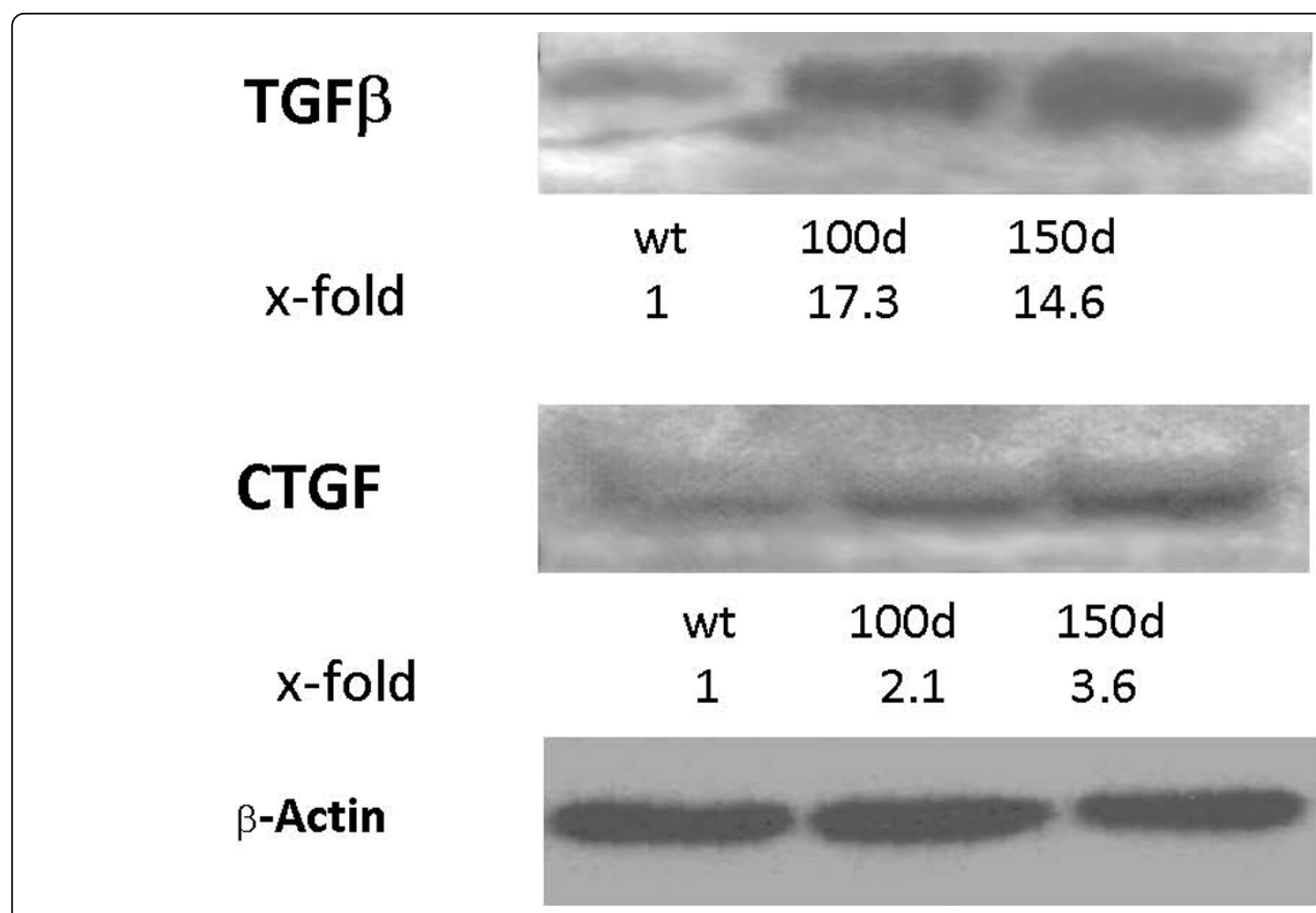

Figure 3 Upregulation of pro-fibrotic factors. Integrin $\alpha 2$ (ITGA2) knockouts showed increased levels of transforming growth factor (TGF) $\beta$ and connective tissue growth factor (CTGF) in whole kidney extracts.

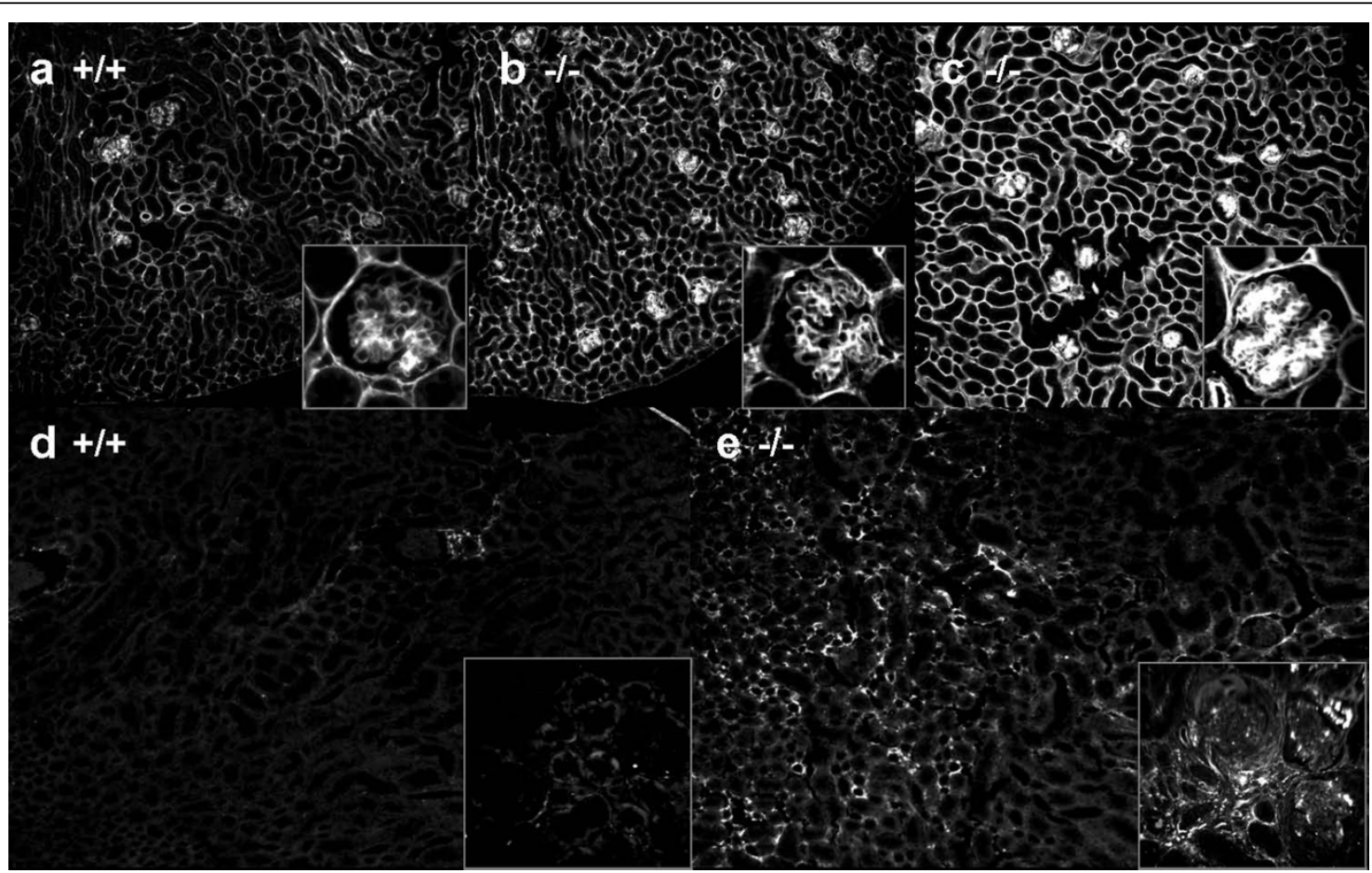

Figure 4 Immunostaining for extracellular matrix and scar tissue. (a-c) EHS laminin; (d, e) fibronectin. Compared with (a) 150-day-old wild-type control mice, (b) 100-day-old and (c) 150-day-old Integrin $\alpha 2$ (ITGA2) knockout mice expressed increased amounts of extracellular matrix. (e) In parallel, 150-day-old ITGA2 knockouts showed accumulation of tubulointerstitial and glomerular scar tissue compared with (d) wild-type mice of the same age. 


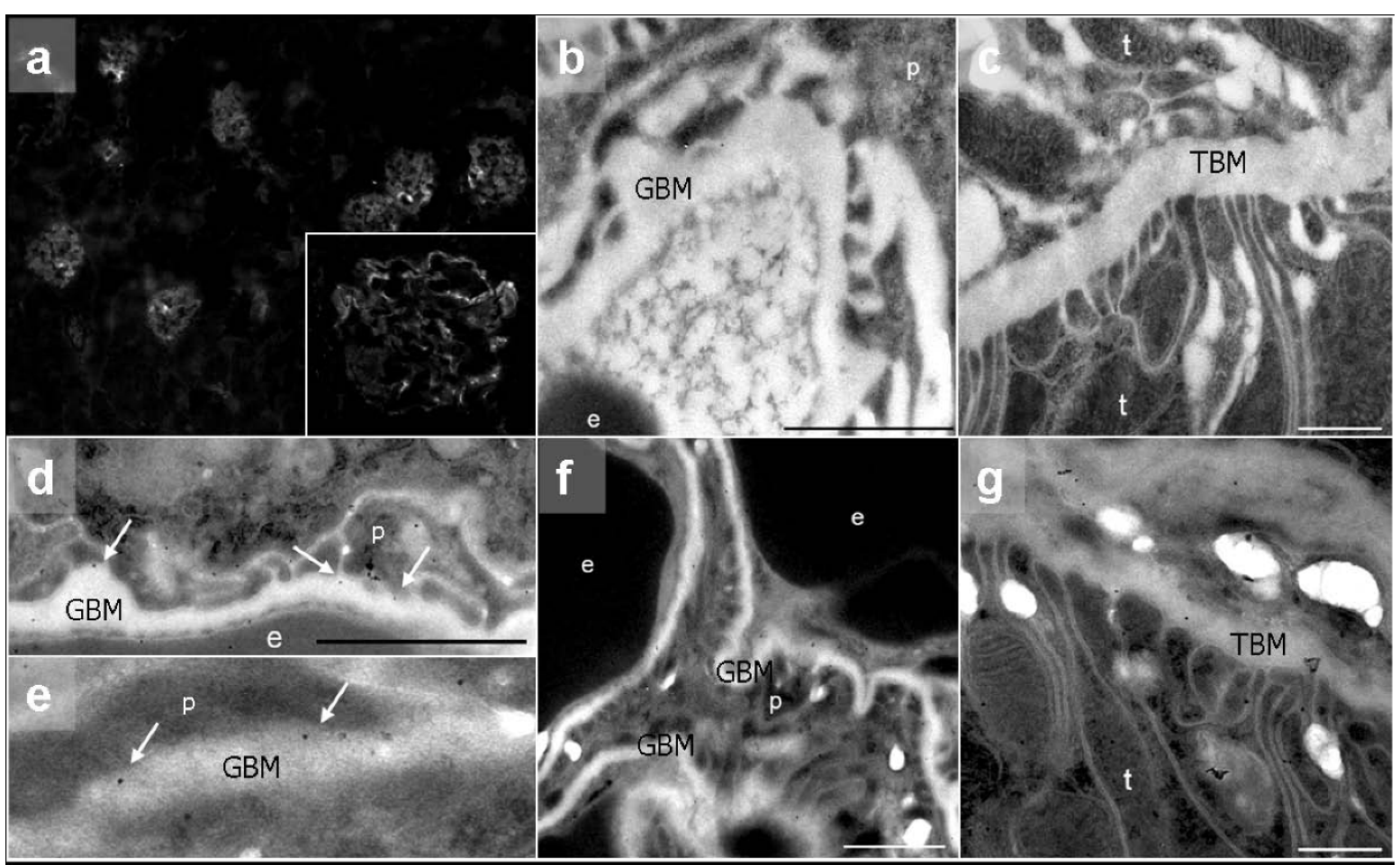

Figure 5 Localization of integrin $\boldsymbol{\alpha 2}$ (ITGA2) expression. (a) Immunohistochemistry of wild-type mice with an antibody to ITGA2 showed staining solely in the glomeruli, whereas kidney tubules remain unstained. (Inset) At higher magnification, ITGA2 was mainly expressed in cells along the basement membrane, most probably podocytes. (d, e) Immunogold localization within the glomerulus demonstrated localization of ITGA2 primarily at the basal side of the podocyte foot processes and close to the glomerular basement membrane (GBM) (white arrows). (d-f) ITGA2 was not detected on the surface of endothelial cells. (b, c) Negative controls in ITGA2 knockouts. Original magnification: (a) $\times 100, \times 400$; (b-g) 20,000 to 30,000. e = erythrocyte; $\mathrm{p}=$ podocyte foot processes; $\mathrm{t}=$ tubular cell; TBM = tubular basement membrane.

fibrosis score (range 0 to 2) was 1.1 at 150 days in ITGA2 knockouts compared with 0.3 in wild-type mice. In parallel, the glomerulosclerosis index (range 0 to 2 ) increased to 0.7 in ITGA2 $2^{-1-}$ mice at 150 days compared with 0.4 in their wild-type littermates.

\section{Localization of integrin $\alpha 2$ expression}

Immunostaining localized integrin $\alpha 2$ solely to the glomeruli, whereas the tubules remained unstained (Figure 5a). At higher magnification, cells sitting on the outer side of the GBM, most probably podocytes, expressed integrin $\alpha 2$ (Figure $5 \mathrm{a}$ insert). These results were further strengthened by immunogold localization: ITGA2 was found primarily at the basal side of the podocyte foot processes close to the GBM (Figure 5d, e, white arrows ). The surface of podocytes always showed staining for ITGA2, but it was never detected on the surface of endothelial cells (Figure 5d-f).

\section{GBM ultrastructural changes result in moderate proteinuria}

Serum urea level of 150-day-old ITGA2 knockout mice was $45.3 \pm 3 \mathrm{mmol} / \mathrm{l}$ and was not significantly increased compared with healthy mice (Figure 6f). Further, mutant mice showed moderate proteinuria (Figure 6a-e), with slightly increased excretion of higher molecular weight proteins in the 150-day-old knockout mice (Figure 6c) compared with healthy controls (Figure 6a, which was in contrast to the severe proteinuria of $\mathrm{COL} 4 \mathrm{~A}^{-1-}$ mice with ongoing renal failure (Figure 6d).

\section{Discussion}

The present study investigated the role of the collagen receptor integrin $\alpha 2$ in the kidney. Confirming our hypothesis, type IV collagen receptors were able to downregulate de novo collagen synthesis as long as the GBM was intact. This hypothesis was strengthened by the renal phenotype of DDR1-knockout mice (Figure 1df) [19]. We were able to demonstrate that the loss of integrin $\alpha 2$ expression induces localized matrix overproduction in the GBM similarly to DDR1 knockouts. These changes lead to moderate proteinuria but are not sufficiently severe to cause further renal damage and eventual renal failure. Interestingly, the loss of ITGA2 mimics the renal phenotype of DDR1 knockouts, indicating a similar role of both collagen receptors within the kidney. 

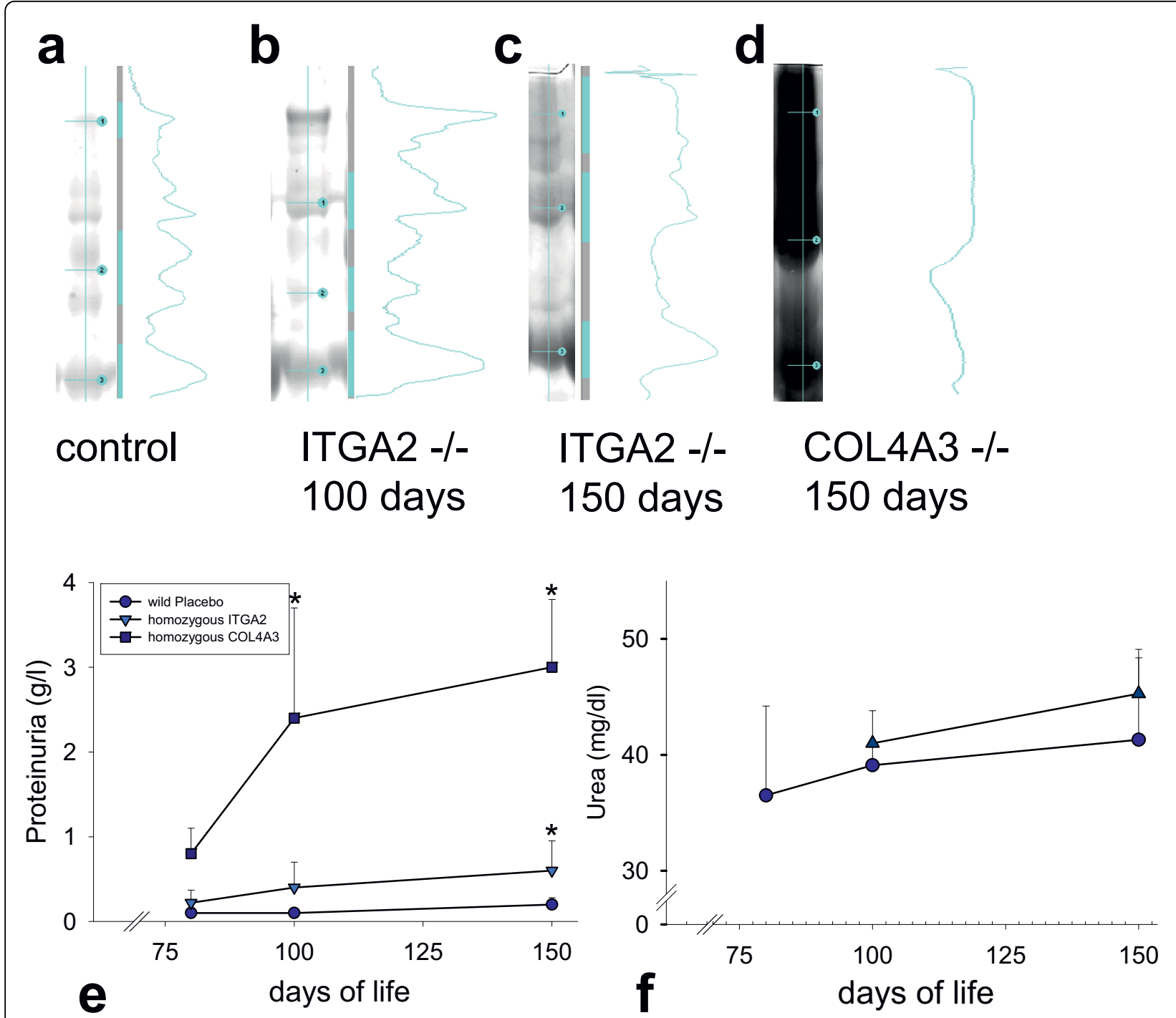

Figure 6 Proteinuria and renal function in integrin $\boldsymbol{\alpha 2}$ (ITGA2) knockouts. Electrophoresis of urine proteins reveals middle- and highmolecular weight proteinuria at (b, c) 100 and 150 days compared with (a) healthy wild-type controls. However, the amount of proteinuria in (e) ITGA2 knockouts (triangles) wa s much lower than in COL4A3 knockouts (with ongoing renal failure and nephrotic syndrome; squares). (f) Levels of serum urea of ITGA2 knockouts (triangles) were not significantly different from those of healthy controls (circles) at 100 and 150 days.

In patients with mutated type IV collagen genes, receptors become unable to recognize collagen correctly. As a consequence, signals suppressing collagen synthesis are depleted, inducing non-physiological overproduction (with faulty collagen chains such as $\alpha 1 / \alpha 2$ type IV collagen chains, fibrillar collagens, and various laminins) [12]. In chronic renal fibrotic diseases such as AS, glomerular and tubulointerstitial matrix deposition and scarring are regulated by TGF $\beta$ and CTGF. The present study shows that loss of the collagen receptor integrin $\alpha 2$ increases the total amounts of renal TGF $\beta$ and CTGF. TGF $\beta$ is known to stimulate the production of
ECM in podocytes [25], thus inducing GBM thickening in the ITGA2 knockouts. These alternations of the GBM lead to proteinuria, which in turn eads to secondary tubular damage and tubulointerstitial fibrosis. However, it remains unclear whether this proteinuria triggers upregulation of TGF $\beta$ and other chemokines or if the global loss of ITGA2 itself leads to tubulointerstitial fibrosis. Future studies should concentrate on the role of ITGA2 in renal disease and on the possible balancing role of both ITGA2 and DDR1.

Within the glomerulus, bioactive TGF- $\beta 1$ is derived from the serum of the capillary lumen or from glomerular 
cells [22]. TGF $\beta$ upregulates type IV collagen in all glomerular cell types [25], and increases promoter activity of fibronectin and $\alpha 2$ type I collagen genes. Further, a consensus TGF- $\beta$ element is located in the promoter of the rat pro $\alpha 1$ type I collagen gene [26]. TGF- $\beta$ induces the expression of CTGF via a functional Smad3 binding site in the CTGF promoter. In diabetic nephropathy, CTGF has been reported to play an important role in glomerular alteration by inducing the production of fibronectin and of collagen types I and IV [27].

According to our hypothesis, podocytes do not only produce mature GBM components ( $\alpha 3 / 4 / 5$ (IV) collagen) [13], but also react to altered GBM composition via collagen receptors, leading to matrix overproduction. Integrin $\alpha 1 \beta 1$ is crucial for downregulating collagen synthesis, suggesting that its functional loss could enhance renal fibrosis [28]. Additionally, in the present study, the localization of integrin $\alpha 2$ at the lateral base of podocytes points to an important role of integrin $\alpha 2$ in the cell-matrix interaction between the GBM and podocytes. ITGA2 seems not to be crucial for embryogenesis. However, cell-matrix interaction via tyrosine kinase receptors such as DDR1 and ITGA2 seems to be very important to the mature kidney, otherwise different collagen receptors compensating each other's role would not exist. Future experiments should focus on the balanced function and interaction of DDR1 and ITGA2.

Abrahamson et al. recently demonstrated the distinct role of the podocyte in the preservation and production of mature GBM components [13]. This group further verified that collagen receptors, particularly integrins, are linked to the cytoskeleton of podocytes. The activation of DDR1 has been shown to influence smooth muscle cell migration and matrix metalloproteinase expression [29]; both mechanisms play an important role in the pathogenesis of AS [5,10,12,30,31]. Future experiments should focus on the role of collagen receptors such as integrin $\alpha 2$ in the architecture and cytoskeleton of podocytes. Further, interaction between the GBM, podocytes and slit membrane might become increasingly important in the pathogenesis of chronic fibrotic renal diseases and the pathogenesis (and therapy) of proteinuria.

Our data provide evidence that altered cell-matrix interaction via collagen receptors such as $\alpha 2$ integrin and DDR1 are one important step in the maintenance and production of ECM and in the sequence of fibrogenesis. Thus, targeting collagen receptors might be important in the treatment of progressive fibrotic diseases.

\section{Conflict of interests statement}

The authors declare that they have no competing interests.

\section{Authors' contributions}

The manuscript was drafted by RG and OG; the study was conceived by MM, JK, NM and JT; the study's design and coordination was done by RG, NMi, BE, G-AM and OG. All authors read and approved the final manuscript.

\section{Acknowledgements}

This study was supported by the German Research Foundation DFG, grant numbers GR 1852/4-1 and 4-2 (to OG). Parts of this work were supported by the DFG through SFB 829 (to B.E.). We would also like to thank Mrs Andrea Bernhard for her excellent technical assistance.

\section{Author details}

'Department of Nephrology and Rheumatology, Georg-August-University Goettingen, Goettingen, Germany. ${ }^{2}$ Department of Prosthodontics, Tissue Regeneration Work Group, University Medicine, Georg-August-University Goettingen, Goettingen, Germany. ${ }^{3}$ Department of Dermatology, University of Cologne, Cologne, Germany.

Received: 31 May 2010 Accepted: 22 September 2010

Published: 22 September 2010

\section{References}

1. Belkin VM, Belkin AM, Koteliansky VE: Human smooth muscle VLA-1 integrin: purification, substrate specificity, localization in aorta, and expression during development. J Cell Biol 1990, 111:2159-2170.

2. Colognato-Pyke H, O'Rear JJ, Yamada Y, Carbonetto S, Cheng YS, Yurchenco PD: Mapping of network-forming, heparin-binding, and alpha 1 beta 1 integrin-recognition sites within the alpha-chain short arm of laminin-1. J Biol Chem 1995, 270:9398-9406.

3. Kreidberg JA, Symons JM: Integrins in kidney development, function, and disease. Am J Physiol Renal Physiol 2000, 279:F233-F242.

4. Gardner H, Broberg A, Pozzi A, Laato M, Heino J: Absence of integrin alpha1beta1 in the mouse causes loss of feedback regulation of collagen synthesis in normal and wounded dermis. J Cell Sci 1999, 112:263-272.

5. Cosgrove D, Rodgers K, Meehan D, Miller C, Bovard K, Gilroy A, Gardner H, Kotelianski V, Gotwals P, Amatucci A, Kalluri R: Integrin alpha1beta1 and transforming growth factor-beta1 play distinct roles in alport glomerular pathogenesis and serve as dual targets for metabolic therapy. Am J Pathol 2000, 157:1649-1659.

6. Kühn K, Eble J: The structural bases of integrin-ligand interactions. Trends Cell Biol 1994, 4:256-261.

7. Chen J, Diacovo TG, Grenache DG, Santoro SA, Zutter MM: The alpha(2) integrin subunit-deficient mouse: a multifaceted phenotype including defects of branching morphogenesis and hemostasis. Am J Pathol 2002, 161:337-344.

8. Yang JT, Rayburn H, Hynes RO: Embryonic mesodermal defects in alpha 5 integrin-deficient mice. Development 1993, 119:1093-1105.

9. Gardner H, Kreidberg J, Koteliansky V, Jaenisch R: Deletion of integrin alpha 1 by homologous recombination permits normal murine development but gives rise to a specific deficit in cell adhesion. Dev Biol 1996, 175:301-313.

10. Gross O, Beirowski B, Koepke ML, Kuck J, Reiner M, Addicks K, Smyth N, Schulze-Lohoff $E$, Weber M: Preemptive ramipril therapy delays renal failure and reduces renal fibrosis in COL4A3-knockout mice with Alport syndrome. Kidney Int 2003, 63:438-446.

11. Miner JH, Sanes JR: Collagen IV alpha 3, alpha 4, and alpha 5 chains in rodent basal laminae: sequence, distribution, association with laminins, and developmental switches. J Cell Biol 1994, 127:879-891.

12. Hudson BG, Tryggvason K, Sundaramoorthy M, Neilson EG: Alport's syndrome, Goodpasture's syndrome, and type IV collagen. N Engl J Med 2003, 348:2543-2556.

13. Abrahamson DR, Hudson BG, Stroganova L, et al: Cellular Origins of Type IV Collagen Networks in Developing Glomeruli. J Am Soc Nephrol 2009, 20:1471-1479.

14. Borza DB, Bondar $\mathrm{O}$, Todd $\mathrm{P}$, et al: Quaternary organization of the goodpasture autoantigen, the alpha 3(IV) collagen chain. Sequestration of two cryptic autoepitopes by intrapromoter interactions with the alpha4 and alpha5 NC1 domains. J Bio/Chem 2002, 277:40075-40083. 
15. Vogel W, Brakebusch C, Fässler R, Alves F, Ruggiero F, Pawson T: Discoidin domain receptor 1 is activated independently of beta(1) integrin. J Biol Chem 2000, 275:5779-5784.

16. Brakebusch C, Bouvard D, Stanchi F, Sakai T, Fässler R: Integrins in invasive growth. J Clin Invest 2002, 109:999-1006.

17. Nakamura T, Miller D, Ruoslahti E, Border WA: Production of extracellular matrix by glomerular epithelial cells is regulated by transforming growth factor-beta 1. Kidney Int 1992, 41:1213-1221.

18. Takeuchi Y, Suzawa M, Kikuchi T, Nishida E, Fujita T, Matsumoto T: Differentiation and transforming growth factor-beta receptor downregulation by collagen-alpha2beta 1 integrin interaction is mediated by focal adhesion kinase and its downstream signals in murine osteoblastic cells. J Biol Chem 1997, 272:29309-29316.

19. Gross O, Beirowski B, Harvey SJ, McFadden C, Chen D, Tam S, Thorner PS, Smyth N, Addicks K, Bloch W, Ninomiya Y, Sado Y, Weber M, Vogel WF: DDR1-deficient mice show localized subepithelial GBM thickening with focal loss of slit diaphragms and proteinuria. Kidney Int 2004, 66:102-11126.

20. Gross O, Girgert R, Beirowski B, Kretzler M, Kang HG, Kruegel J, Miosge N, Busse AC, Segerer S, Vogel WF, Müller GA, Weber M: Loss of collagenreceptor DDR1 delays renal fibrosis in hereditary type IV collagen disease. Matrix Biol 2010, 29(5):346-56.

21. Holtkötter O, Nieswandt B, Smyth N, Müller W, Hafner M, Schulte V, Krieg T, Eckes B: Integrin alpha 2-deficient mice develop normally, are fertile, but display partially defective platelet interaction with collagen. $J$ Biol Chem 2002, 277:10789-10794.

22. Hong SW, Isono M, Chen S, Iglesias-De La Cruz MC, Han DC, Ziyadeh FN: Increased glomerular and tubular expression of transforming growth factor-beta1, its type II receptor, and activation of the Smad signaling pathway in the $\mathrm{db} / \mathrm{db}$ mouse. Am J Pathol 2001, 158:1653-1663.

23. Miosge N, Sasaki T, Timpl R: Angiogenesis inhibitor endostatin is a distinct component of elastic fibers in vessel walls. FASEB J 1999, 13:1743-1750.

24. Kruegel J, Sadowski B, Miosge N: Nidogen-1 and nidogen-2 in healthy human cartilage and in late-stage osteoarthritis cartilage. Arthritis Rheum 2008, 58:1422-1432.

25. Zeisberg M, Ericksen MB, Hamano Y, Neilson EG, Ziyadeh F, Kalluri R: Differential expression of type IV collagen isoforms in rat glomerular endothelial and mesangial cells. Biochem Biophys Res Commun 2002, 295:401-407.

26. Meisler NT, Chiu JF, Cutroneo KR: Promoter competitors as novel antifibrotics that inhibit transforming growth factor-beta induction of collagen and noncollagen protein synthesis in fibroblasts. $J$ Cell Biochem 1999, 75:196-205.

27. Connolly SB, Sadlier D, Kieran NE, et al: Transcriptome profiling and the pathogenesis of diabetic complications. J Am Soc Nephrol 2003, 14: S279-S283.

28. Sakharova OV, Taal MW, Brenner BM: Pathogenesis of diabetic nephropathy: focus on transforming growth factor-beta and connective tissue growth factor. Curr Opin Nephrol Hypertens 2001, 10:727-738.

29. Hou G, Vogel WF, Bendeck MP: Tyrosine kinase activity of discoidin domain receptor 1 is necessary for smooth muscle cell migration and matrix metalloproteinase expression. Cirches 2002, 90:1147-1149.

30. Cosgrove D, Meehan DT, Grunkemeyer JA, et al: Collagen COL4A3 knockout: a mouse model for autosomal Alport syndrome. Genes Dev 1996, 10:2981-2992.

31. Ronco $P$, Chatziantoniou C: Matrix metalloproteinases and matrix receptors in progression and reversal of kidney disease: therapeutic perspectives. Kidney Int 2008, 74:873-878.

doi:10.1186/1755-1536-3-19

Cite this article as: Girgert et al: Integrin $\alpha 2$-deficient mice provide insights into specific functions of collagen receptors in the kidney. Fibrogenesis \& Tissue Repair 2010 3:19.

\section{Submit your next manuscript to BioMed Central and take full advantage of:}

- Convenient online submission

- Thorough peer review

- No space constraints or color figure charges

- Immediate publication on acceptance

- Inclusion in PubMed, CAS, Scopus and Google Scholar

- Research which is freely available for redistribution

Submit your manuscript at www.biomedcentral.com/submit
C Biomed Central 